\title{
Improved Tool and Data Selection in Task Management
}

\author{
John W. Hagerman and Stephen W. Director \\ Department of Electrical and Computer Engineering \\ Carnegie Mellon University, Pittsburgh, PA 15213
}

\begin{abstract}
Task management involves task creation and execution. These are facilitated using a task schema as exemplified in the Hercules Task Manager. Experience with Hercules has shown the task schema to be very useful for task creation, but less than ideal for task resolution, i.e., the selection of tool and data resources to be used in execution. Tool/data interactions often lead to resource selection constraints that cannot be captured using dependency relationships in the schema. We have addressed this by adding conditions to the task schema which use task-level meta-data to constrain resource selection. With examples we show that conditions are useful for handling a wide variety of real constraints.
\end{abstract}

\section{Introduction}

Task management is an important feature of design frameworks. A task is a set of steps leading from one set of data to another. For example, a task in the EDA domain might be to synthesize a circuit from a specification and then to use simulation to estimate circuit performance. Task management involves task creation (building a task tree), task resolution (selecting tools and data), and task execution (running the tools).

One approach to task management is to use a task schema which captures the relationships among tools and data. This approach is taken by the Hercules Task Manager [1]. Through Hercules we have found the task schema approach to be very useful in a wide variety of design situations. However, we have also found that the task schema does not handle the subtleties of task resolution very well. Specifically, the interactions among tools and data that must be considered during resolution often cannot be captured as simple dependency relationships. This is subtle because although the interactions can be captured as relationships, the relationships are not of the kind used in the original task schema formulation. We have addressed this by enhancing the task schema with the addition of conditions, which are Boolean expressions that use information attached to tools and data to constrain selection. In this paper we describe how we added conditions to the Odyssey framework [2] and show how conditions can be used to capture the constraints needed for tool ordering enforcement, multi-function tool management, tool/data matching, and data coherence.

We begin with a review of the Odyssey framework, Hercules, and task schemata. Then we describe the addition of conditions to Hercules, including the changes made to the task-level database, the schema, the resource manager, and the Hercules User Interface. Then we present results, and close with conclusions.

\section{Background}

Odyssey is a design framework test-bed developed at Carnegie Mellon University. In Odyssey, framework responsibilities are handled in a layered manner, as shown in Figure 1. Hercules is the task management layer. Tool and data details such as file locations and formats are hidden from Hercules by the Cyclops resource manager [3]. Tool and data resources are encapsulated, and Cyclops presents a uniform view of resources to Hercules. Thus, Cyclops mediates between encapsulations and Hercules.

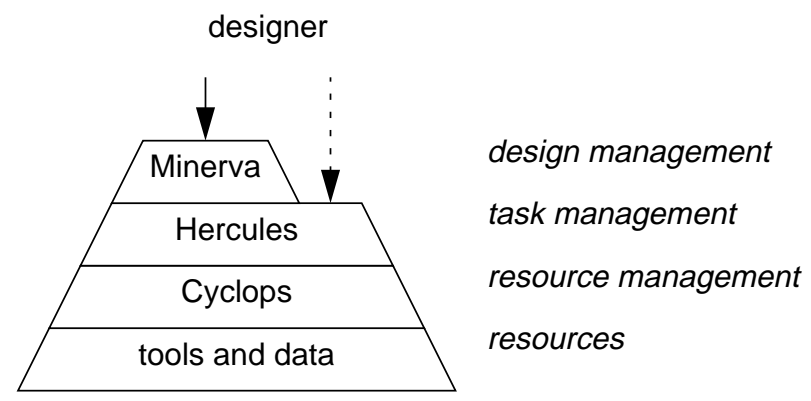

Figure 1: The Layers of the Odyssey Framework

A designer can interact directly with Hercules to manage tasks. Hercules also provides task management to the Minerva design process management layer currently under development [4]. The designer gives problem specifications and goals to Minerva, and Minerva helps the designer break the problem into sub-problems and map them onto tasks. This paper focuses on Hercules.

\subsection{The Task Schema}

In order to manage tasks, a framework must be aware of the relationships among tools and data. At the task level the unnecessary distinction between tools and data is removed by representing all resources as entities. (This is important for the accurate modeling of entities such as scripts that act as tools at some times and data at other times.) A hierarchy of entity classes (types) is defined. Each actual resource is an instance of some class. A task schema is an entity-relationship graph: vertices represent classes, and directed edges represent dependencies. An edge $X \rightarrow Y$ means that $\mathrm{X}$ depends on $\mathrm{Y}$, that is, an instance of $\mathrm{Y}$ is needed to make an instance of $X$. The Hercules database holds static and dynamic information. The class hierarchy and the schema are static, while information about instances is dynamic. Information about an instance includes the mapping to the Cyclops encapsulation, and other meta-data such as creation time and creator username.

Consider the schema shown in Figure 3. Entity classes are drawn as boxes. Classes are hierarchical: a generic class is specialized to capture the methods by which instances are generated. In the figure, the generic class specification has two specializations: analyzed-spec and transformed-spec. The edges indicate, for example, that an evaluation has a functional dependence (" $\mathrm{f}$ ") on an evaluator and a data dependence ("d") on a specification. Circular data dependencies may exist from a specialization to its generic class; two are shown in the figure.

A task is easily created from a schema. A task tree has vertices for entity classes and edges to indicate information flow. The task tree in Figure 5 could have been created from the schema of Figure 3. The root is at the bottom, and it should be clear how tree edges are obtained from schema dependencies. The goal of a task is to create an instance of the entity at the root of the tree; all the other vertices represent the tools and data to be used to achieve the goal. (Odyssey allows multiple roots; we will assume a single root and mention multiple roots only as necessary.) or distributed for profit or commercial advantage, the copyright notice, the title of the publication and its date appear, and notice is given that copying is by permission of ACM, Inc. To copy otherwise, or to republish, to post on servers or to redistribute to lists, requires prior specific permission and/or a fee. DAC 96 - 06/96 Las Vegas, NV, USA 


\subsection{Task Resolution}

Tasks created from schemas are abstract, i.e., they are defined in terms of entity classes rather than specific instances. Therefore, before a task can be executed, specific tool and data instances to be used in the execution must be selected. This process is called task resolution. When instances have been selected for all leaves of a task tree the task is resolved. To execute a resolved task, the framework invokes the tools in an order that creates instances for the interior vertices as necessary to achieve the goal.

Task resolution is performed as follows. Figure 5 is the Hercules User Interface display of a task tree. At the top of each vertex box is the entity class name, and at the bottom is the selected instance name, if an instance has been selected. In the figure, an instance has been selected only for the transformer vertex. The user chooses a vertex to resolve and a browser appears which lists all the selectable instances for that vertex. The user selects an instance from the list, or selects no instance, and clicks an OK button. The user continues in this way, resolving vertices one at a time. When the task is resolved the user can request that it be run.

\section{Selection Constraints}

Experience with Hercules has shown that the task schema is very useful for creating tasks. We have also found, however, that tool/ data interactions often imply resource selection constraints that cannot be captured using dependencies. For example, consider the analysis/transformation design flow shown in Figure 2. First, a specification is evaluated to determine whether it meets a set of goals. If the goals are met then design can proceed. Otherwise the specification is analyzed to determine what changes are necessary. The analysis tool annotates the specification to record the needed changes, and the transformation tool bases its operation on the annotations. Then the evaluation is repeated.

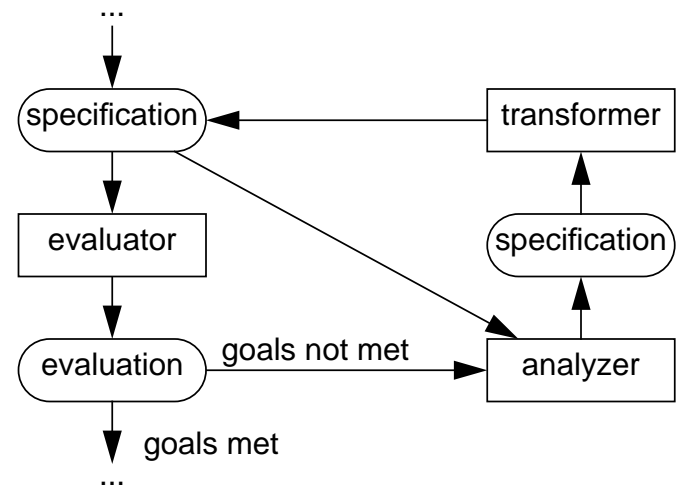

Figure 2: An Analysis/Transformation Design Flow

The data passed among the tools is all of the same type: a specification. Thus, the task schema illustrated in Figure 3 accurately captures the relationships among the tools and data.

This task schema indicates that a new specification is obtained by applying either an analysis or a transformation tool to an existing specification. However, an important relationship not captured in the schema is that analysis must be performed before transformation, since analysis adds information to the specification that is used in transformation. Thus we must track whether or not each specification instance has annotation information. We do this by attaching attribute meta-data to the instances, where an attribute is a name-value pair. Here we use an annot attribute to indicate whether a specification instance has been annotated. Then we add conditions to the task schema to test the attribute, as shown in Figure 4. The condition ! ? annot @ 2 on the analyzed-spec
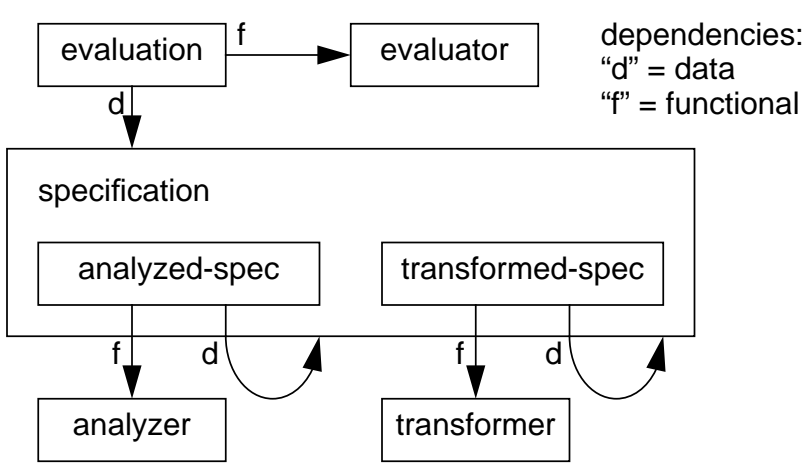

Figure 3: Analysis/Transformation Task Schema

entity means that specification instances at the other end of edge number 2 ("d2" in the figure) must not have the annot attribute to be selectable (! ? is the "not-exist" operator). The condition ? ? annot \& 4 on transformed-spec means that specification instances at the other end of edge number 4 ("d4" in the figure) must have the attribute to be selectable. This shows the flexibility of using conditions to enforce orderings. The transformed-spec condition specifies only that the specification have an annot attribute. How and when that attribute is created is of no concern to the transformation tool, so this method permits any sequence of steps that leads to a properly annotated specification.

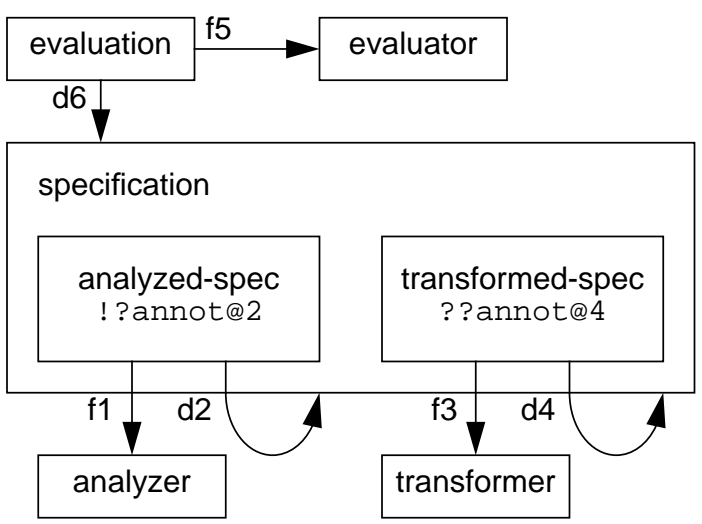

Figure 4: Previous Schema with Conditions Added

\subsection{Condition Evaluation}

In general, a condition is a Boolean combination of literals that test attributes attached to instances so as to constrain selection. Condition evaluation occurs as follows. The selection state of a task indicates the selection of instances at all vertices. It is with respect to this state that conditions are evaluated. For example, suppose the user is resolving the task of Figure 5, created from the schema of Figure 4, and suppose that the user has chosen to resolve the specification vertex. When resolving a tree vertex, it is the conditions at the parent of the vertex that must be satisfied. The parent of the tree vertex specification is analyzed-spec, which has the condition ! ?annot @2. To generate the list of selectable instances for the specification vertex, each appropriate instance is proposed in turn as being selected, the selection state is updated to reflect the proposal, and the parent's condition is evaluated with respect to that state. (In a tree with multiple roots, some vertices will have multiple parents, and the conditions of all parents are evaluated.) The instance is added to the list being generated, along with a record of the condition evaluation result. If the condition failed then text describing the failure is stored 
(see Section 4.3). After the list is generated, the user chooses instances as usual. When the user chooses an instance for which a condition failed, the stored text is used to generate feedback.

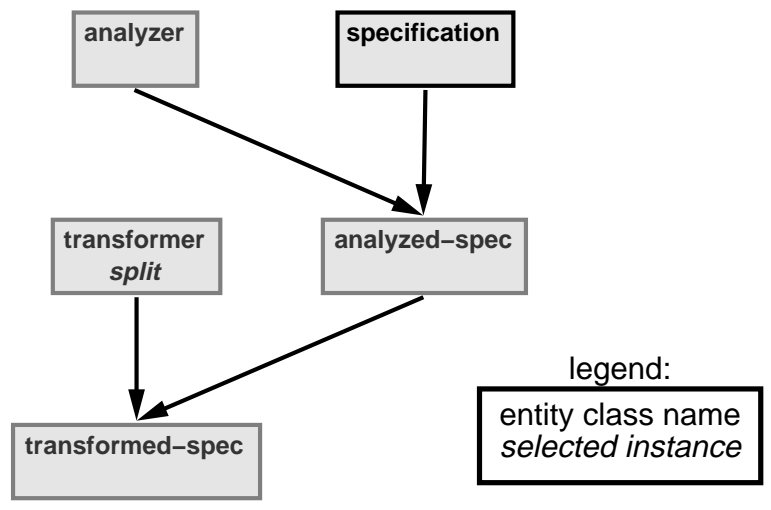

Figure 5: Hercules User Interface View of a Task Tree

There are two special cases involving missing instance selections for siblings of the vertex being resolved. The first case is where the sibling is a leaf, as when resolving the specification vertex in Figure 5, since no instance is selected for the analyzer leaf. To illustrate, assume that the analyzed-spec condition is changed to ! ?annot@2 \&\& ??annotator@1, where an annotator attribute is attached to analyzer tool instances which annotate specifications. Even though no instance is selected for analyzer, it must be possible to evaluate the condition when resolving the specification vertex. Also, it is important not to over-constrain the list of instances. These requirements are met using lenient evaluation: condition literals that refer to unknowns evaluate to true, and thus those literals are effectively removed.

The second case occurs when an interior sibling has no selected instance. This will be the case when resolving the transformer vertex in Figure 5, since no instance is selected for the analyzedspec vertex. It must be possible to evaluate the transformedspec condition, and the evaluation must not be over-constraining. Again, these requirements are satisfied using lenient evaluation.

Note that no instance needs to be selected for the analyzed-spec vertex for the task to become resolved. When the task is run, the analyzer tool will be invoked first to create a new analyzed-spec instance, and then the transformer tool will be invoked to create a new transformed-spec instance using the new analyzed-spec instance. Since a lenient evaluation of the transformed-spec condition was performed, the condition must be evaluated again after creating the new analyzed-spec instance to check that the condition still holds - if it does not, then task execution must be aborted, and condition failure feedback must be given to the user.

\section{Implementation}

Changes were needed in the task database, the Cyclops encapsulation methods, the task schema, and the Hercules User Interface.

\subsection{Task Database Changes}

The main change to the task database was the addition of general attributes. Attributes have string values, there are no restrictions such as "instances of this entity class must have these attributes," and strings are interpreted as numbers in numeric contexts.

\subsection{Cyclops Changes}

Attributes are meta-data for instances, with semantic meaning due to conditions. Thus, attribute creation must be considered carefully. There are two possibilities: attributes may be created by the task manager or by the tools. It might be possible for the task manager to create attributes in some cases. For example, for the schema in Figure 4 it might suffice for the task manager to attach annot attributes to new analyzed-spec instances. However, this will not work for tools such as editors, for which it may be impossible to know what attributes should be attached to new instances. It is better to have the tools (or, more specifically, the tool encapsulations) create attributes. This requires changes to Cyclops. When Hercules invokes a tool, Cyclops executes the tool's encapsulation, and the encapsulation runs the tool. When the tool finishes, the encapsulation sends completion information back to Cyclops. We have added the ability for the encapsulation to send back a set of attributes for the new instance. This, then, is a very general way to capture attribute creation semantics in the framework, since encapsulations are "in" the framework.

\subsection{Task Schema Changes}

Above we stated that conditions are Boolean combinations of literals that test attributes. In fact, conditions are restricted to a sum-of-products form, as indicated by this pseudo-syntax:

$$
\begin{aligned}
\text { condition } & ::=\text { term } \| \text { term } \| \ldots \\
\text { term } & ::=\text { literal } \mathbf{\&} \boldsymbol{\&} \text { literal } \mathbf{\&} \ldots \\
\text { literal } & ::=\text { exist-op attr-deref } \\
& ::=\text { str-operand str-op str-operand } \\
& :=\text { num-operand num-op num-operand } \\
\text { exist-op } & ::=\mathbf{?}, ! ? \\
\text { str-op } & ::=\mathbf{e q}, \mathbf{n e}, \mathbf{g t}, \mathbf{g e}, \mathbf{l t}, \mathbf{l e} \\
\text { num-op } & ::===, !=,>,>=,<= \\
\text { str-operand } & ::=\mathbf{N A M E} @ \text { number } \\
& ::=\mathbf{U S E R} @ \text { number } \\
& ::=\text { attr-deref } \\
& ::=\text { string } \\
& ::=\text { attr-deref } \\
::= & \text { number } \\
\text { num-operand } & ::=\text { name@ } @ \text { number }
\end{aligned}
$$

Attributes are accessed using the name@number syntax. Edges leaving an entity class are numbered so they can be identified, as shown in Figure 4. The name@ number syntax means "the value of attribute name on the instance at the end of the edge numbered number." Built-in meta-data is accessed using special names: NAME for instance name and USER for creator username.

The sum-of-products (SOP) form has two advantages. First, it enables the simple generation of condition failure messages. An English description of the failure of a literal is easy to generate, and the SOP form makes it easy to produce a description for an entire condition (e.g., "Condition failed for parent analyzed-spec: ! ?annot (2"). Allowing general Boolean expressions would make failure isolation hard. Secondly, the SOP form enables the useful semantics of lenient evaluation, in that a literal that refers to an unknown can affect only one term.

\subsection{User Interface Changes}

Conditions cause instance selection at one vertex to be affected by selections at other vertices, so users should be allowed to see all instances, even those that cause condition failure. If the user tries to select such an instance then a feedback message is given which describes the condition failure and selection is rejected.

\section{Results}

The example of Figure 4 showed how conditions are useful for 
order enforcement. This example is also useful for illustrating the management of multiple tool instances. The real transformation tool of interest performs one of two operations, split or merge, so there are two encapsulations (and thus instances) of the tool. It is possible to define a single transformer class for both instances, even though they have different input requirements: split requires the specification to be annotated, but merge can be performed on any specification. This is captured using conditions as illustrated in Figure 6. A split attribute is attached to the split instance of the transformer tool. Then the condition on transformed-spec ! ?splite3 || ? ?annot@ 4 means that either the merge tool is used (i.e., no constraint on the input specification) or, if the split tool is used, the input specification must be annotated. This allows the designer to define a "transformation" task, where the selection of the specific operation is deferred to task execution time, with tool input restrictions enforced by the condition.

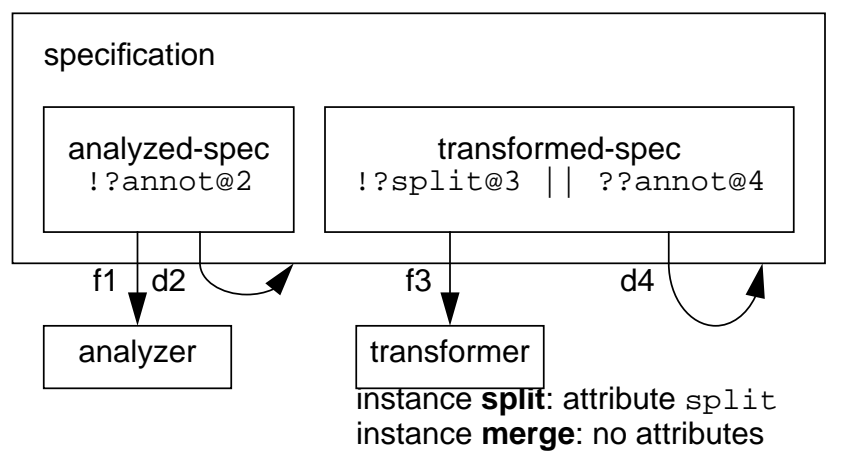

Figure 6: One Tool Class, Multiple Tool Functions

Conditions can be used to ensure that tools and data "match." This is illustrated by the example in Figure 7, where two circuitsimulator tools support different input format "levels": HSPICE supports input extensions not known by SPICE. This is handled by attaching a lev attribute to all circuit-simulator and netlist instances, where the attribute value indicates the input format level supported by the tool: 1 for spice and 2 for hspice. Then the condition lev@ $2<=$ lev@ 1 constrains selection so that the tool is able to handle the data. In the figure, the selection of the spice tool and the low pow data will cause the condition to fail.

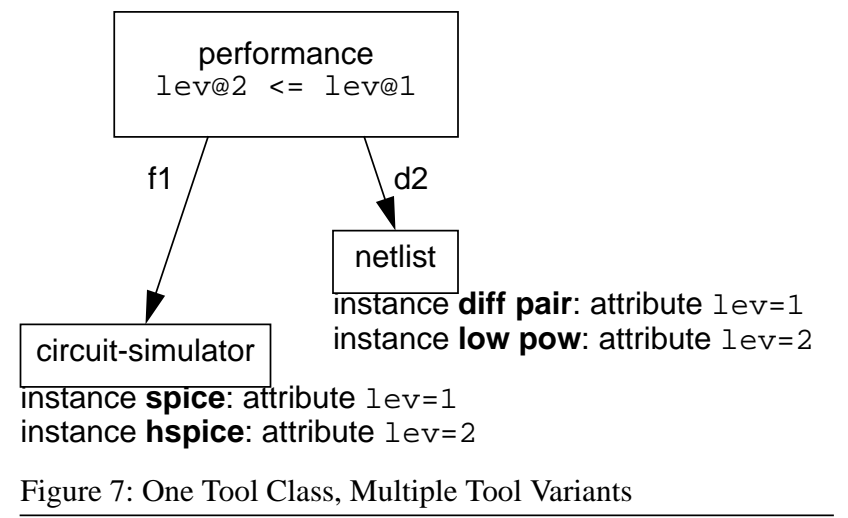

Another example involves "data coherence." The design flow in Figure 8 shows the behavioral synthesis tasks of scheduling and allocation. The alloc tool data inputs must be coherent, i.e., the control flow must have been derived from the value trace.

A simple method of ensuring data coherence using conditions is illustrated in Figure 9. A ver attribute is attached to all the data

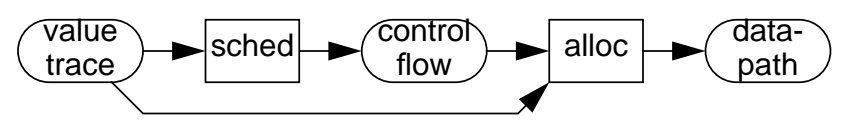

Figure 8: A Design Flow Requiring Data Coherence

instances to keep track of "versions." The scheduler tool encapsulation copies the version from the input value trace to the output control flow, and the condition ver@ $1==$ ver@ 2 ensures that the data instance inputs to the allocator tool are coherent.

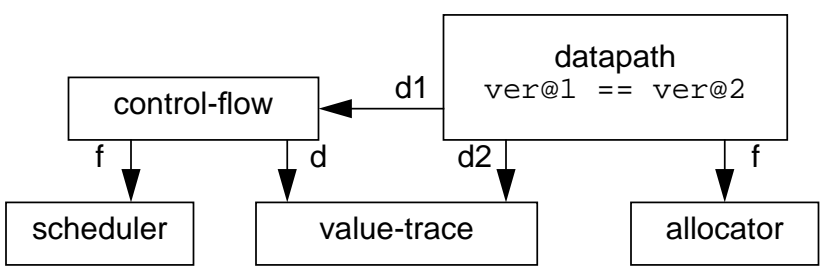

Figure 9: A Task Schema to Ensure Data Coherence

Finally, conditions are more general than the similarly-motivated capabilities described in [5]. The constraints described therein are tightly tied to the framework semantics, and are thus likely to have difficulty handling unanticipated constraint needs.

\section{Conclusion}

The task schema concept as originally embodied in the Hercules Task Manager was good for task creation but insufficient for task resolution, because resource selection constraints often cannot be captured as dependency relationships. We have overcome this by adding to task schemas conditions which test instance meta-data. Selection is constrained by evaluating the conditions for each proposed selection. Using a sum-of-products form ensures that instance selection is not overly constrained, and allows the easy generation of English feedback descriptions of condition failures. The result is a good balance of power and generality: conditions provide graceful solutions to real problems, and are also easy to use and understand.

\section{Acknowledgments}

The authors would like to thank the reviewers for their detailed and helpful comments. This research was supported in part by the Semiconductor Research Corporation, contract DC-068.

\section{References}

[1] J.B. Brockman and S.W. Director, "The Schema-Based Approach to Workflow Management," IEEE Trans. on $C A D$, vol. 14, no. 10, October 1995, pp. 1257-67.

[2] J.B. Brockman et al., "The Odyssey CAD Framework," DATC Newsletter on Design Automation, Spring 1992.

[3] T.F. Cobourn, "Resource Management for CAD Frameworks," Ph.D. dissertation, Carnegie Mellon University, July 1992, Research Report No. CMUCAD-92-39.

[4] M.F. Jacome and S.W. Director, "A Formal Basis for Design Process Planning and Management," Int. Conf. on Computer-Aided Design, November 1994, pp. 516-21.

[5] P. van der Wolf, O. ten Bosch, and A. van der Hoeven, "An Enhanced Flow Model for Constraint Handling in Hierarchical Multi-View Design Environments," Int. Conf. on Computer-Aided Design, November 1994, pp. 500-7. 